\title{
Simultaneously suppressing frequency and intensity noise in a Nd:YAG nonplanar ring oscillator by means of the current-lock technique
}

\author{
Michèle Heurs, Volker M. Quetschke, ${ }^{*}$ Benno Willke, and Karsten Danzmann \\ Max-Planck-Institut für Gravitationsphysik (Albert-Einstein-Institut) and Institut für Atom- und Molekülphysik, Abteilung \\ Spektroskopie, Universität Hannover, Callinstrasse 38, D-30167 Hannover, Germany \\ Ingo Freitag \\ InnoLight GmbH, Garbsener Landstrasse 10, D-30419 Hannover, Germany
}

Received April 6, 2004

\begin{abstract}
We show that frequency and intensity noise in a Nd:YAG laser are correlated to a high degree and can be traced to the same underlying cause, namely, power fluctuations of the pump source. Because of this correlation, simultaneous suppression of frequency and intensity noise by $30 \mathrm{~dB}$ is achieved by means of a single actuator, the pump power. (c) 2004 Optical Society of America

OCIS codes: $140.3580,140.3570$.
\end{abstract}

High-precision metrology experiments, such as interferometric gravitational-wave detectors, ${ }^{1-4}$ require ultrahigh-stability laser light sources. Monolithic laser-diode-pumped Nd:YAG nonplanar ring oscillators (NPROs) offer high intrinsic stability in frequency, intensity, and beam geometry ${ }^{5,6}$ and are used as master lasers for injection-locked laser systems in various interferometric gravitational-wave detectors. ${ }^{1,7,8}$ Yet to achieve the required ultrahigh sensitivity for these detectors the laser systems have to be further actively stabilized.

The intensity noise of a NPRO can be actively suppressed by means of a noise eater, a circuit that feeds back to the current of the pump laser diodes. ${ }^{9}$ Conventional frequency stabilization of a NPRO to a rigid cavity in vacuum makes use of one or more actuators, usually a piezoelectric transducer (PZT) in contact with the NPRO crystal for fast changes and the temperature of the crystal for slow changes. Using the pump current as an actuator for frequency stabilization is an alternative approach referred to as current lock. ${ }^{10}$ This method shows various advantages over using the PZT as an actuator; it does not introduce excess beam pointing and shows no resonances up to the resonant relaxation oscillations (RROs).

Using the pump current to tune the frequency of an NPRO is bound to cause a worsening of intensity noise in the NPRO. Instead it was experimentally shown by our group ${ }^{10}$ that current lock resulted in a small amount $(3 \mathrm{~dB})$ of intensity noise suppression in small bandwidth. This observation spawned completely new experiments described in the following, because it is desirable to attain a stronger intensity noise suppression as the by-product of frequency stabilization by means of the current-lock technique. This is not possible in a conventional NPRO, however. The multimode laser diode array, which is the common NPRO pump source, exhibits strong spatial and temporal mode fluctuations, even when the pump current is held extremely stable. These mode fluctuations, even when the pump current is held extremely stable. These mode fluctuations cause intensity noise as well as frequency noise in the NPRO, and both noise processes are uncorrelated. We therefore constructed an NPRO pumped by a single-mode laser diode (SM-NPRO). Here the pump mode is spatially and temporally unvarying. Stabilization of the pump current of a single-mode diode can even result in squeezing of the pump light ${ }^{11}$; in our case it leads to a simultaneous stabilization of the NPRO intensity and frequency noise, because both noise processes are strongly correlated in the SM-NPRO.

The principle of the current-lock technique is the coupling of pump power modulations into the output power as well as into the laser frequency. On the one hand, pump power fluctuations couple directly into fluctuations of the output power of the NPRO at Fourier frequencies well below the RRO. ${ }^{12}$ On the other hand, pump power variations are also an important noise source for frequency fluctuations of the $\mathrm{NPRO}^{13}$ : Pump power modulation leads to modulation of the deposited thermal energy in the active medium. This thermal modulation changes the index of refraction of the material as well as the length of the crystal; both phenomena result in a change of the optical path length in the Nd:YAG material. Hence any optical path-length modulation causes a frequency modulation of the laser light. As the crystal acts as a thermal low-pass filter, the pump current modulation decreases with increasing modulation frequency, and the transfer function of frequency change per current modulation shows the typical $1 / f$ behavior. ${ }^{10,14}$

However, for frequencies approaching the RRO of the NPRO another effect becomes dominant in the transfer function. Pump power modulation leads to modulation in the inversion of the active medium and therefore to variations in the index of refraction, which entails frequency modulation of the laser light. This effect is a small perturbation to the steady state but is clearly 
visible in the above-mentioned transfer function at the RRO frequency. Efforts toward an understanding of this effect are under way.

In a NPRO with a multimode pump source the frequency noise suppression from current lock is very high (more than $100 \mathrm{~dB}$ at $100 \mathrm{~Hz}$ ), and the corresponding intensity noise suppression is nearly negligible. ${ }^{10}$ To increase the degree of simultaneous intensity and frequency noise suppression in current lock, we constructed a SM-NPRO in cooperation with InnoLight $\mathrm{GmbH}$. It consists of a single-mode laser diode (SDL/JDS Uniphase, type 5422-H1-810), wavelength nominally $810 \mathrm{~nm},{ }^{15}$ which is fiber-coupled into a polarization-maintaining single-mode fiber. The pump module of the SM-NPRO including beamshaping optics is constructed on a Microbank system (Schäfter + Kirchhoff). The pump light with a power of approximately $70 \mathrm{~mW}$ after the fiber is matched to the fundamental mode of a NPRO crystal by an optical telescope. The NPRO, designed for low threshold, then emits approximately $10 \mathrm{~mW}$ of laser light at $1064 \mathrm{~nm}$. Figure 1 shows the schematic setup of the SM-NPRO.

In the experiment described below we stabilized the SM-NPRO frequency to the resonance frequency of a rigid reference cavity by use of the current-lock technique to further investigate the effect of frequency and intensity noise coupling in this system.

A rigid high-finesse three-mirror ring resonator (reference cavity) was placed in an ultrahigh vacuum (pressure $p \approx 10^{-7} \mathrm{~Pa}$ ) as a frequency reference for the laser system. Another ring resonator with a piezoelectric actuator for frequency tuning was placed in a different vacuum chamber and served as an independent reference (analyzer cavity) for frequency noise measurements. In both cases the Pound-Drever-Hall technique was used to stabilize the laser frequency to the reference cavity (unity gain frequency $\approx 4 \mathrm{kHz}$ ) and the analyzer cavity length to the laser frequency (unity gain frequency $\approx 2 \mathrm{kHz}$ ). The laser light was split by a power beam splitter and individually mode matched into the two cavities. The frequency of the SM-NPRO was stabilized to the reference cavity by means of the current-lock technique. Below unity gain the feedback signal of this control loop is proportional to the free-running frequency noise of the laser relative to the reference cavity. The error signal of this control loop yields the information on the free-running frequency noise of the laser above the unity gain frequency and the in-loop frequency noise reduction below unity gain.

The analyzer cavity was then stabilized to the SM-NPRO by feeding back to its PZT, and thereby the analyzer cavity resonance frequency was locked to the laser frequency by means of the Pound-DreverHall method. The feedback and error signals of this control loop contain the information on the independently measured (out-of-loop) frequency noise of the SM-NPRO, when it is stabilized to the reference cavity. Below the unity gain frequency of the analyzer cavity control loop the feedback signal to the PZT of the analyzer cavity is a direct measure of the remaining out-of-loop laser frequency noise relative to the reference cavity. Above the unity gain of this loop the error signal can be used as a redundant measure for the free-running frequency noise of the laser. The finesse of the analzyer cavity is $F=4440 .{ }^{16}$ With the round-trip length of the analzyer cavity being $l_{\mathrm{rt}}=42 \mathrm{~cm}$, the free spectral range (FSR) according to $\mathrm{FSR}=c / l_{\mathrm{rt}}$ is $714 \mathrm{MHz}$. The corresponding linewidth $\Delta \nu$ (half-width at half-maximum) is therefore $80 \mathrm{kHz}$ [determined by $F=\mathrm{FRS} /(2 \Delta \nu)$ ], so that it does not act as an optical low pass at the detection frequencies. A photodetector placed behind a pickoff plate in the laser beam simultaneously measures the intensity noise of the free-running and the frequency-stabilized SM-NPRO. The control scheme is displayed in Fig. 2.

Current lock of the SM-NPRO to the reference cavity leads to a strong in-loop frequency noise reduction of the laser with a maximum suppression of more than $60 \mathrm{~dB}$ at $10 \mathrm{~Hz}$. This in-loop frequency noise reduction is limited by the gain of the control loop locking the laser by means of current lock to the reference cavity, not by detection noise. A measurement of the frequency noise with the independent analyzer cavity shows that the out-of-loop frequency noise is suppressed by approximately $30 \mathrm{~dB}$. At low Fourier frequencies the out-of-loop frequency noise is limited by detection noise; for Fourier frequencies of approximately $100 \mathrm{~Hz}$ the suppression is gain limited. Both in- and out-of-loop measurements of the frequency noise of the SM-NPRO are shown in Fig. 3.

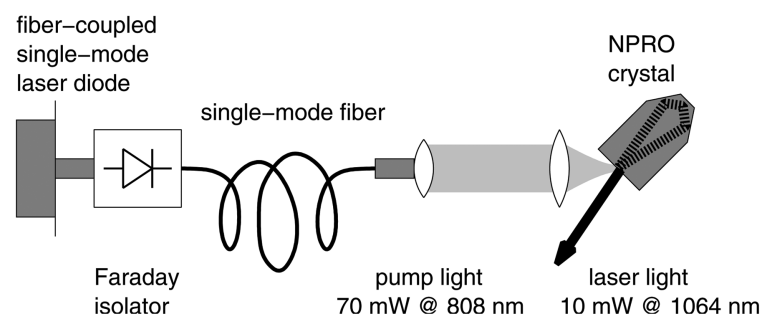

Fig. 1. Schematic of the single-mode laser-diode-pumped NPRO.

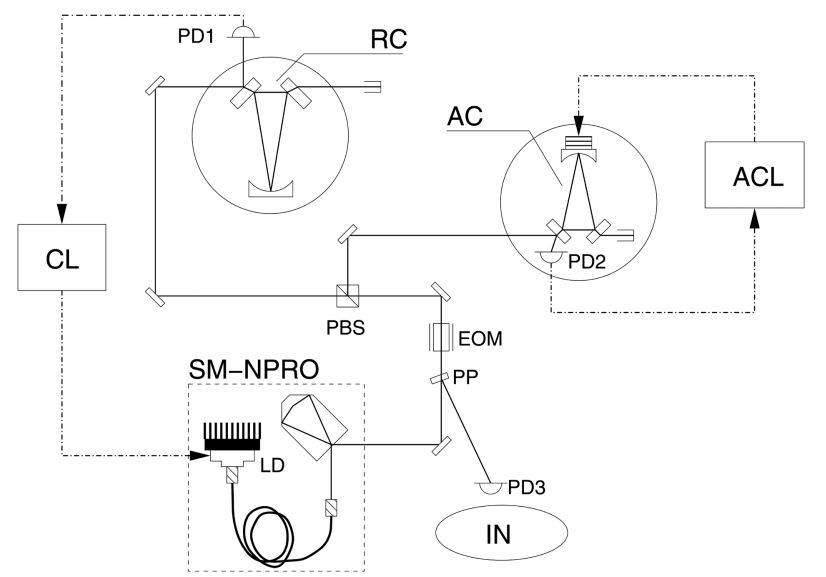

Fig. 2. Stabilization scheme for the single-mode laserdiode-pumped NPRO. PD1-PD3, photodetectors; RC, reference cavity; AC, analyzer cavity; CL, current lock stabilization SM-NPRO to reference cavity; ACL, stabilization analyzer cavity to SM-NPRO; PBS, polarizing beam splitter; EOM, electro-optic modulator; PP, pickoff plate; LD, laser diode; IN, intensity noise measurement. 


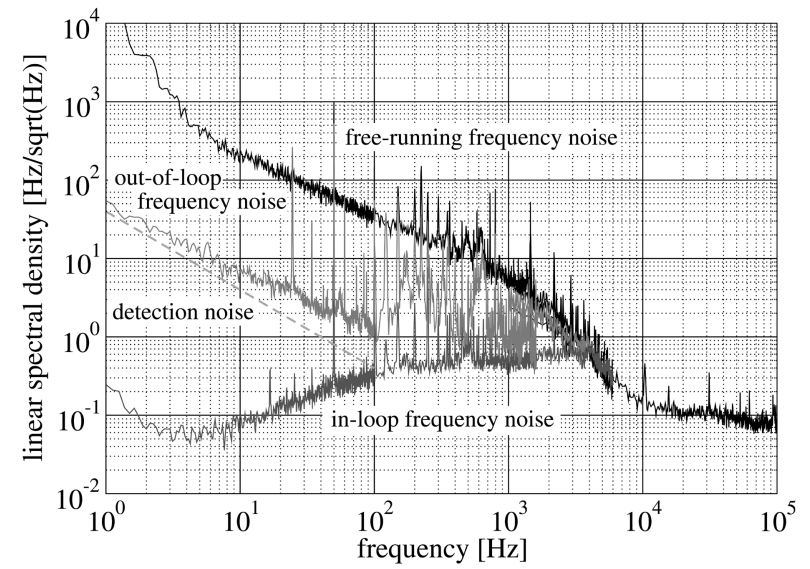

Fig. 3. Free-running, in-loop, and out-of-loop frequency noise of the SM-NPRO with current lock.

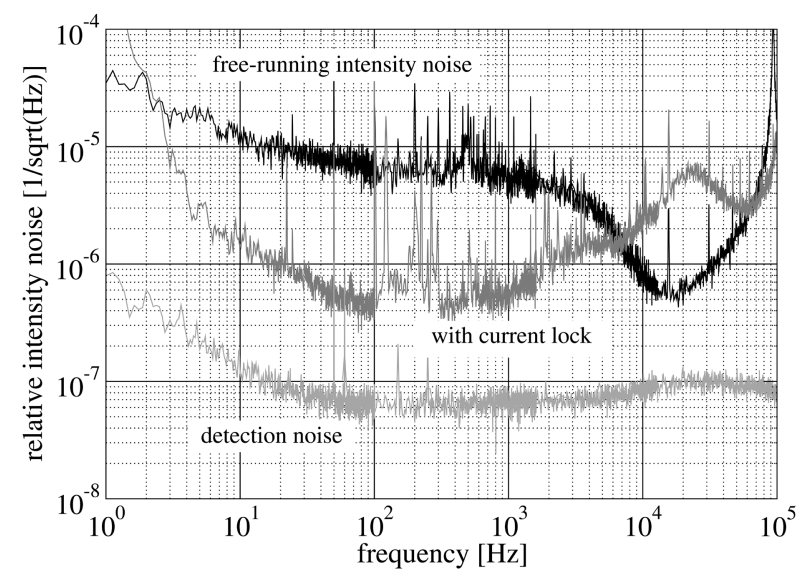

Fig. 4. Intensity noise of the SM-NPRO with and without current lock

The intensity noise of the SM-NPRO is measured with a photodetector behind a pickoff plate. Figure 4 shows the free-runnning intensity noise of the laser as well as the intensity noise of the laser when it is frequency stabilized to the reference cavity by means of current lock. It is obvious that the free-running intensity noise is suppressed in current lock by the same amount as the out-of-loop frequency noise, relative to the free-running frequency noise (for low Fourier frequencies $1 / f$ flicker noise dominates the spectrum and limits the suppression). This intensity noise suppression is achieved without any further actuation, even without the detection of intensity noise in the first place. It is indeed a positive effect of the coupling of intensity and frequency noise in the NPRO, as described in the previous section.

We have demonstrated simultaneous frequency and intensity noise suppression of $30 \mathrm{~dB}$ in a single-mode laser-diode-pumped monolithic Nd:YAG NPRO. This was achieved by frequency stabilization of the NPRO to a rigid cavity through feedback to the pump current (current lock). Because of the strong noise correlation, simultaneous intensity stabilization resulted without further actuation.
Furthermore, it is interesting to know whether intensity stabilization of the pump source has an effect on the intensity noise or even on the frequency noise of the NPRO, as in a conventional NPRO pump intensity stabilization that does not improve the intensity noise characteristics of the NPRO. The experiments were successfully conducted by our group. ${ }^{17}$

Using a master oscillator power amplifier system (single-mode laser diode and amplifier stage with an output power of approximately $500 \mathrm{~mW}$ ) as a pump source promises higher NPRO output power of more than $200 \mathrm{~mW}$, while being able to use the described technique to simultaneously suppress intensity and frequency noise. This NPRO can then be used as a master laser for an injection-locked laser system for gravitational-wave detection.

M. Heurs's e-mail address is michele.heurs@ aei.mpg.de.

*Currently at the University of Florida, Gainesville, Florida 32611.

\section{References}

1. B. Willke and the GEO 600-Team, Class. Quantum Grav. 21, 417 (2004).

2. F. Acernese and the VIRGO collaboration, Class. Quantum Grav. 21, 385 (2004).

3. M. Ando and the TAMA collaboration, Class. Quantum Grav. 19, 1409 (2002).

4. B. Abbott and the LIGO Science Collaboration, Nucl. Instrum. Methods Phys. Res. A 517, 154 (2004).

5. T. Kane and R. Byer, Opt. Lett. 10,65 (1985).

6. I. Freitag, A. Tünnermann, and H. Welling, Opt. Commum. 115, 511 (1995).

7. S. T. Yang, Y. Imai, M. Oka, N. Eguchi, and S. Kubota, Opt. Lett. 21, 1676 (1996).

8. F. Cleva, M. Taubman, C. N. Man, and A. Brillet, in Gravitational Waves, E. Coccia, G. Veneziano, and G. Pizzella, eds. (World Scientific, Singapore, 1998), pp. $321-327$.

9. C. C. Harb, M. B. Gray, H.-A. Bachor, R. Schilling, P. Rottengatter, I. Freitag, and H. Welling, IEEE J. Quantum Electron. 30, 2907 (1994).

10. B. Willke, O. S. Brozek, K. Danzmann, V. Quetschke, and S. Gossler, Opt. Lett. 25, 1019 (2000).

11. Y. Yamamoto, S. Machida, and O. Nilsson, Phys. Rev. A 34, 4025 (1986).

12. C. C. Harb, T. C. Ralph, E. H. Huntington, D. E. McClelland, H.-A. Bachor, and I. Freitag, J. Opt. Soc. Am. B 14, 2936 (1997).

13. O. Svelto, Principles of Lasers, 4th ed. (Plenum, New York, 1998), p. 293.

14. V. M. Quetschke, "Korrelationen von Rauschquellen bei Nd:YAG Lasersystemen," Ph.D. dissertation (Universität Hannover, Hannover, Germany, 2003).

15. "SDL-5400 Series 50 to $200 \mathrm{~mW}, 810 / 830 / 852 \mathrm{~nm}$ Single-mode Laser Diodes" (JDS Uniphase, San Jose, Calif., 2002), http://www.jdsu.com/site/ images/products/pdf/5400_010302.pdf.

16. B. Willke, N. Uehara, E. K. Gustafson, R. L. Byer, P. J. King, S. U. Seel, and R. L. Savage, Jr., Opt. Lett. 23, 1704 (1998).

17. M. Heurs, V. M. Quetschke, B. Willke, I. Freitag, and K. Danzmann are preparing a paper detailing these experiments. 